Case Report

\title{
Cervical external root resorption: 3-year follow-up of a case
}

\author{
Dimitris Nikolidakis, George Nikou, Gert J. Meijer and John A. Jansen \\ Department of Periodontology and Biomaterials, Radboud University Nijmegen Medical Center, \\ Nijmegen, Netherlands
}

(Received 1 May and accepted 27 August 2008)

\begin{abstract}
Cervical external root resorption can be present in natural teeth, involving one or more teeth in the same patient. The incidence of these lesions appears random and the etiology remains unclear. Cervical external root resorption has been considered a difficult clinical situation, and its diagnosis and treatment of the defect challenging. The present report describes a case of multiple external cervical resorption lesions involving four teeth, including the history, and the clinical and radiographic findings. The treatment included surgical intervention and restoration of the defect without sacrificing the pulp. A 3-year reevaluation of the case confirmed a stable, uneventful clinical recovery. (J. Oral Sci. 50, 487-491, 2008)
\end{abstract}

Keywords: external root resorption; cervical lesion; case report.

\section{Introduction}

Localized lesions of root resorption can be present in natural teeth, mainly in the apical and mid-region of the root (1). The etiology of both internal and external root resorption remains unclear, and may occur in the absence of either local or systemic factors. External resorption is a process that may lead to loss of cementum, dentin and bone. It takes place in both vital and non-vital teeth, and is mostly identified during routine radiographic or clinical examination, as the majority of cases are asymptomatic.

Correspondence to Dr. Dimitris Nikolidakis, Department of Periodontology \& Biomaterials, Radboud University Nijmegen Medical Center, PO Box 9101, 6500 HB Nijmegen, The Netherlands

Tel: +31-24-3614920

Fax: +31-24-3614657

E-mail: D.Nikolidakis@dent.umcn.nl
External resorption may be physiological or pathological, and several investigators have classified it into various categories $(2,3)$. Consequently, there is still a lack of uniformity in the literature, which is confusing for the dental practitioner. External root resorption has been found to be an infrequent phenomenon that affects either the apical or cervical region of one or several teeth. It is relatively rare to find idiopathic external resorption associated with cervical areas of the tooth and even more uncommon for the condition to involve multiple teeth.

The first reported case of cervical root resorption was described by Mueller and Rony in 1930 (4). Cervical external resorption has also been called invasive cervical resorption (5) or peripheral inflammatory root resorption (6) and represents a special type of inflammatory resorption. In recent years, several etiologic factors have been advocated and some morphological descriptions have appeared. Nevertheless, prediction and prevention are still impossible, and precise diagnosis and treatment are often far from easy, depending on the severity and localization of the defect. Cervical external resorption occurs immediately below the epithelial attachment of the tooth. As a result, it must be noted that the location is not always cervical but related to the level of the marginal tissues and the pocket depth. Unless proper treatment is initiated, this type of resorption continues, and a large irreversible loss of tooth structure may appear in time.

Clinically, cervical external resorption is associated with inflammation of periodontal tissues and does not involve the pulp (7). The resorptive cavities are usually shallow and confined to the cementum layer without penetrating deeply into the underlying dentin, the pulp usually remaining protected by a thin layer of dentin until late in the process. Such lesions are likely to be associated with trauma, orthodontic treatment and periodontal disease and usually do not progress rapidly. On the other hand, some 
reports have also described severe external cervical root resorption of unknown etiology (8). In this type of idiopathic resorption, multiple teeth may be involved in both dental arches, lesions often forming at the same time and at about the same rate without primary involvement of the pulp $(9,10)$. The resorption lesions may extend far into the coronal dentin and can occasionally progress rapidly. In some cases, extraction of the affected teeth is the only adequate treatment (11).

The present report describes a case of extensive and multiple cervical root resorption occurring within a period of one year, including details of the history, examination, diagnosis and therapy.

\section{Patient Intake}

A 46-year old Caucasian non-smoking male was referred

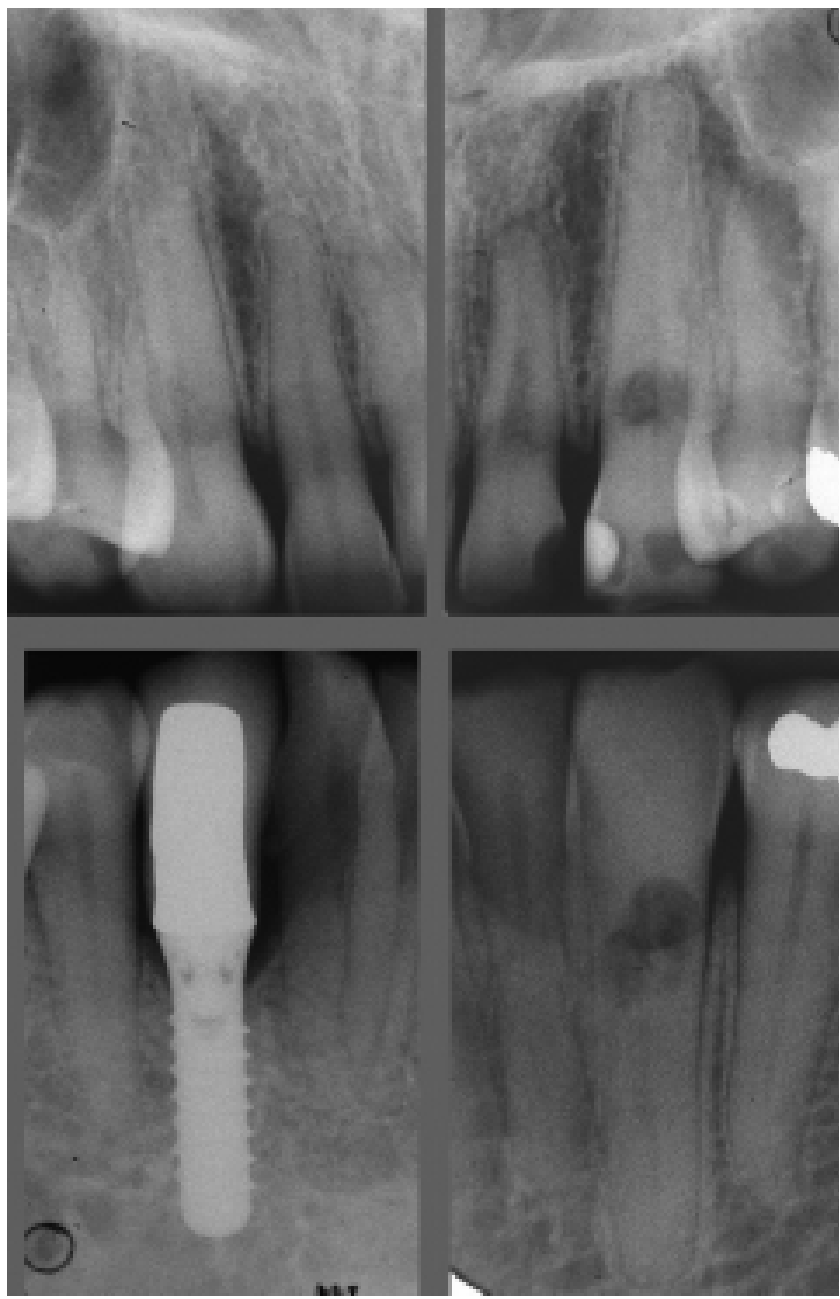

Fig 1. Overview of periapical intraoral radiographs of the canine regions at presentation. Circumferential radiolucent spots are present in the cervical region of teeth \#13, \#23 and \#33. Tooth \#43 has been replaced by an implant. to the Department of Periodontology, Radboud University Nijmegen Medical Centre, in 2003 after his dentist had discovered an unusual lesion in the buccal cervical region of the lower left canine (\#33) on routine radiographic examination. The patient was in good general health without any systemic disease, and had no subjective dental complaints.

\section{Dental history}

The patient had undergone surgical removal of the wisdom teeth in childhood. Also, multiple crowns and amalgam restorations had been placed. In 2001, root resorption in the lower right canine (\#43) had been discovered radiographically after routine recall, and endodontic treatment and prosthetic restoration had been performed. In 2002, the same tooth had been fractured during chewing. Therefore, the dentist removed the tooth and replaced it with an osseointegrated implant. In 2003, root resorption was observed radiographically in tooth \#33, and the patient was referred to our department. He had parafunctional habits (clenching and grinding). His brother also had this problem, and had been provided with a full mouth prosthetic reconstruction due to excessive tooth wear. The patient described himself as having a high degree of work-related stress.

\section{Clinical and radiographic examination}

Oral inspection and evaluation of the patient's periodontal status revealed reasonable oral hygiene, and supragingival calculus was present in the lingual anterior region. The bleeding score upon gentle probing was low, and probing pocket depths of up to $4 \mathrm{~mm}$ were recorded. Localized gum recession was noticed, and occlusal tooth wear and cervical abfractions were also observed. Since the patient did not have any periodontal defects, microbiological testing was not carried out, and so no information about the microflora was obtained. Radiographic examination showed circumscribed radiolucent lesions in the cervical region of teeth \#13,\#23 and \#33, but no periapical pathology was evident in any of the affected teeth. An overview of fullmouth periapical intra-oral radiographs at presentation is presented in Fig. 1. By using a caries explorer, a subgingival concavity on the buccal cervical aspect of tooth \#23 and the buccal and lingual aspect of tooth \#33 was revealed, but tooth \#13 had no clinical sign of tooth surface abnormality. Vitality was positive for all canines, and there was no pain or excessive mobility of these teeth. Evaluation of the dentist's archived radiographic plates showed that the radiolucent cervical lesions in the area of the canines had been present since at least 2001. 


\section{Diagnosis}

Based on the patient's history, and the clinical and radiographic examinations, the diagnosis was generalized gingivitis with external cervical root resorption. The root resorption was characterized as external because the pulp in the root canal was not involved, as the radiopaque mineralized outline of the canal was clearly distinct through the radiolucency of the resorption defects. Also, tooth vitality remained positive throughout the follow-up period. Histological examination of gingival tissue biopsied from the area of the lesion confirmed this diagnosis and revealed inflamed vascular connective tissue infiltrated by lymphocytes. The presence of multinucleated giant cells similar to osteoclasts indicated resorptive activity (12).

\section{Treatment}

The definitive treatment plan included oral hygiene reinforcement, supragingival debridement, construction of a night-guard to relieve the patient of the negative effects of his parafunctional habits and prevent additional tooth wear, and finally surgical intervention for removal of the inflamed granulation tissue that occupied the lesion cavity and repair of the resorption defect with a restoration material. A three-month recall program for supportive periodontal therapy was proposed, with the intention of re-evaluating the case after a long follow-up period.

After the initial non-surgical part of the therapy, a surgical phase (Fig. 2) was introduced. Following flap elevation, the inflamed granulation tissue that occupied the resorption cavity was removed and prepared for histological examination. Invasion of bone into the defect was observed. Ostectomy was performed to expose the cervical defect, and the alveolar crest was extended by contouring to a level $2 \mathrm{~mm}$ apical to the defect margin (13). The cavity was debrided, shaped and filled with glass-ionomer cement (GC Fuji II ${ }^{\circledR}$, Tokyo Japan) according to the method of Heithersay (14). Three resorption cavities were restored: the buccal cervical region of \#23, and the buccal and lingual cervical regions of \#33.

After suturing, postoperative care was applied. Postsurgical pain and edema were controlled with 600-mg ibuprofen tablets, and the patient was instructed to rinse twice daily with $0.12 \%$ chlorhexidine and to use modified oral hygiene procedures in the treated area for the first 4 postoperative weeks. Gentle wiping of the treated dentogingival area with a surgical toothbrush without interdental cleaning was used during the first 4 postoperative weeks. Sutures were removed after 1 week. Post-surgical controls and professional tooth cleaning consisting of supragingival prophylaxis with a rubber cup and $0.2 \%$ chlorhexidine gel were performed at weeks 1, 2, 4 and 6.
During the postoperative sessions, uneventful healing was observed without any extensive pain, hematoma, suppuration or flap dehiscence. The patient was maintained under a supportive care program and received full-mouth professional prophylaxis and calculus removal every 3 months.

\section{Reevaluation}

Six months later, a reevaluation of the case was performed. The general periodontal status was stable with shallow probing pocket depths and reduced bleeding scores. A good level of plaque control (30\% plaque score) was maintained. The patient became accustomed to the night-guard, and no problems were reported. The periodontal probing depth measurements did not reveal any significant changes in the areas of teeth \#13, \#23 and \#33 (ppd $\leq 3 \mathrm{~mm}$ ). Additionally, recessions of the gingival margin around the treated teeth had led to supragingival displacement of the restored defects. Careful clinical examination of tooth \#13 did not reveal any cavity around the cervical region despite the stable radiolucent cervical sign evident on the periapical radiograph. After discussion with the patient, no exploratory surgical procedure was performed, and further follow-up of the case was decided. Radiographic examination confirmed a stable periodontal situation and no deterioration of the cervical defects (Fig. 3 ). No ongoing resorption in the remaining teeth was observed, except for a stable radiolucent spot in the cervical
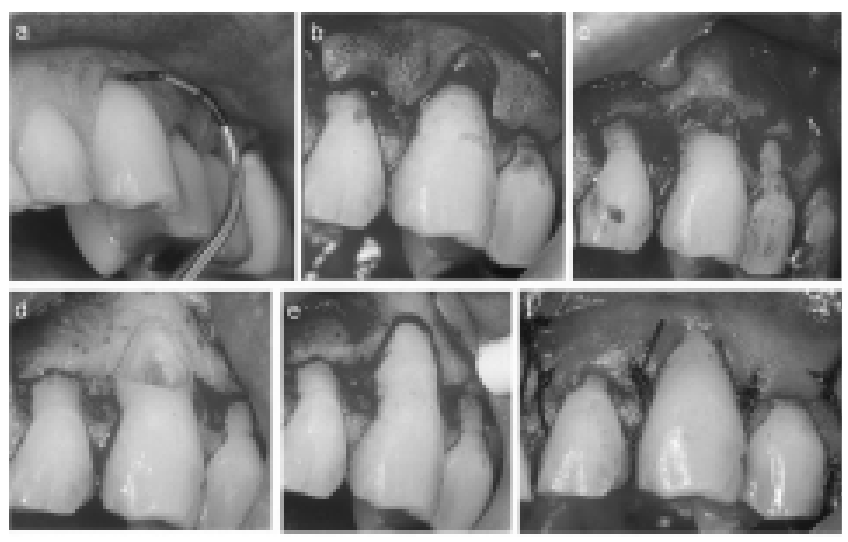

Fig 2. Overview of the surgical approach for tooth \#23: (a) Clinical examination revealed a defect of $3 \mathrm{~mm}$ depth, perpendicular to the cervical surface. (b) Flap design included excision of the pathological granulation tissue that occupied the resorption cavity. (c) After exposure of the defect, invasion of bone into the defect was observed. (d) Ostectomy was performed for cleaning the tooth cavity. (e) After cleaning and shaping, the cavity was filled with glass-ionomer cement. (f) Sutures were applied. 
region of tooth \#13.

Clinical and radiographic re-examination at 3 years after treatment indicated a stable periodontal situation, without further deterioration of the cervical defects.

\section{Discussion}

Diagnosis of cervical external root resorption is made only rarely, but when present it is a challenge for clinicians. The precise etiology of the cervical resorption is still unknown, and many related factors have been proposed. Except for the systemic and idiopathic form, external cervical resorption can occur late after orthodontic tooth movement, oral surgery, periodontal root scaling and planing, tooth bleaching, trauma, bruxism, tooth fracture, developmental defects or a combination of these predisposing factors (3). It has been speculated that it is the result of local damage or alteration of the cervical aspect of the root surface, rendering it susceptible to multinuclear cells with resorptive-clastic activity during an inflammatory response of the periodontal ligament to traumatic or bacterial stimulus (6). It has also been suggested that the periodontal ligament and cementum may play a preventive role, offering resistance to resorption of the root surface (15). In addition, it has been shown that damage to the hard tissue barrier can trigger osteoclastic activity (16), and perhaps a similar phenomenon occurs in the case of resorption defects on the root surface. In the present case, the only factor that could be correlated with these lesions was the patient's bruxism.

Regarding the treatment of external root resorption, successful management of each case must be linked to the etiology. Besides extraction, different approaches have been suggested by several authors for the treatment of cervical external root resorption. Among them, subgingival curettage has been proposed, but this has a high failure rate due to recurrence (5). Some authors have reported the benefit of neutralization the lesion environment by application of calcium hydroxide to the defects (17). In

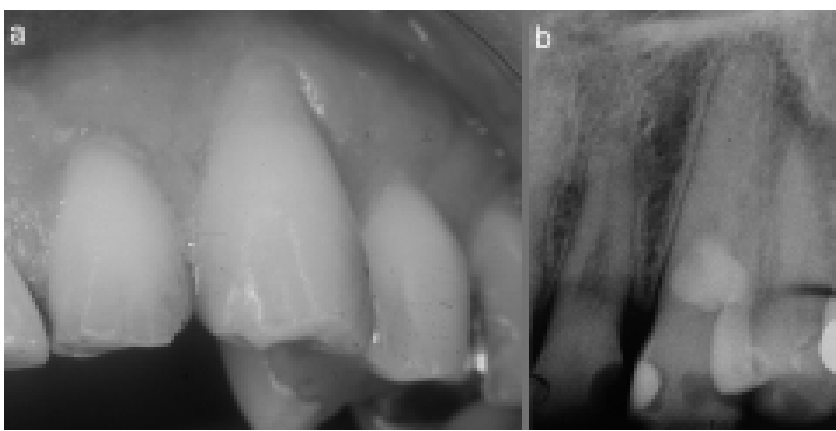

Fig 3. Clinical view (a) and periapical intraoral radiograph (b) of tooth \#23 at the 3-year re-evaluation. another case report (18), the treatment consisted of mechanical debridement supported by systemic antibiotics and dietary advice. A surgical approach with exposure of the resorption defect and ostectomy has also been advocated (13). In our patient, the treatment involved removal of pathologic tissue from the defect and restoration of the resorption cavity. We also considered the management of the patient's parafunctional habit.

It is important for clinicians to consider that this type of lesion may not be an endodontic problem. Careful clinical and radiographic examination of each case can exclude pulp involvement and establish a treatment approach characterized by retaining the vitality of the pulp.

The present case involved multiple external cervical resorption lesions affecting 3-4 teeth, and all the available information related to the case was considered. The treatment approach included surgical intervention, and the final results after a 3-year re-evaluation interval appeared satisfactory.

\section{References}

1. Henry JL, Weinmann JP (1951) The pattern of resorption and repair of human cementum. J Am Dent Assoc 42, 270-290

2. Andreasen JO (1985) External root resorption: its implications in dental traumatology, paedodontics, periodontics, orthodontics and endodontics. Int Endod J 18, 109-118

3. Tronstad L (1988) Root resorption - etiology, terminology and clinical manifestations. Endod Dent Traumatol 4, 241-252

4. Mueller E, Rony HR (1930) Laboratory studies of an unusual case of resorption. J Am Dent Assoc 17, 326-334

5. Heithersay GS (1985) Clinical endodontic and surgical management of tooth and associated bone resorption. Int Endod J 18, 72-92

6. Gold SI, Hasselgren G (1992) Peripheral inflammatory root resorption. A review of the literature with case reports. J Clin Periodontol 19, 523-534

7. Frank AL, Torabinejad M (1998) Diagnosis and treatment of extracanal invasive resorption. J Endod 7, 500-504

8. Moody GH, Muir KF (1991) Multiple idiopathic root resorption. A case report and discussion of pathogenesis. J Clin Periodontol 18, 577-580

9. Kerr DA, Courtney RM, Burkes EJ (1970) Multiple idiopathic root resorption. Oral Surg Oral Med Oral Pathol 29, 552-565 
10. Hopkins R, Adams D (1979) Multiple idiopathic resorption of the teeth. Br Dent J 146, 309-312

11. Liang H, Burkes EJ, Frederiksen NL (2003) Multiple idiopathic cervical root resorption: systematic review and report of four cases. Dentomaxillofac Radiol 32, 150-155

12. Dragoo MR, Sullivan HC (1973) A clinical and histological evaluation of autogenous iliac bone grafts in humans. Part II. External root resorption. J Periodontol 44, 614-625

13. Meister F Jr, Haasch GC, Cernstein H (1986) Treatment of external resorption by a combined endodontic-periodontic procedure. J Endod 12, 542545

14. Heithersay GS (1999) Clinical, radiographic and histopathological features of invasive cervical resorption. Quintessence Int 30, 27-37

15. Lindskog S, Hammarström L (1980) Evidence in favor of an anti-invasion factor in cementum or periodontal membrane of human teeth. Scand J Dent Res 88, 161-163

16. Chambers TJ (1981) Phagocytic recognition of bone by macrophages. J Pathol 135, 1-7

17. Whitworth J (2004) Dental root resorption Part 1. Classification, etiology and management of the external inflammatory lesion. Endod Prac 9, 7-13

18. Beertsen W, Piscaer M, Van Winkelhoff AJ, Everts V (2001) Generalized cervical root resorption associated with periodontal disease. J Clin Periodontol 28, 1067-1073 